\title{
Changes in children's intakes and sources of free sugars since 1997
}

\author{
S.A. Gibson ${ }^{1}$, L.E. Francis ${ }^{1}$, K.J. Newens ${ }^{2}$ and M.B.E. Livingstone ${ }^{3}$ \\ ${ }^{1}$ Sig-Nurture Ltd, Guildford, Surrey, \\ ${ }^{2}$ Sugar Nutrition UK, London, UK and \\ ${ }^{3}$ University of Ulster, $U K$
}

Meeting the target of 5\% energy from free sugars is highly challenging given that present intakes are three times this level in children ${ }^{(1)}$. Understanding the trends in intake may help in designing more effective strategies for sugars reduction. Free sugars intakes and sources among children aged 4-18 y were compared between 1997 NDNS (n1688) and NDNS 2008-2012 (n1687) by analysing original dietary records obtained from the UK Data Service https://www.ukdataservice.ac.uk/. Non-milk extrinsic sugars (NMES) was used as proxy for free sugars. Means were compared using non-parametric (Mann Whitney) tests.

Mean intakes of free sugars (\% energy) among 4-18y olds fell by 10\% (1.7 percentage points) between 1997 and 2008-2012 (mean $16.8 \%$ vs. $15.1 \%$ ) $\mathrm{P}<0.001$ ), with a larger fall of $15 \%$ among $4-10 \mathrm{y}$ olds (mean $17.4 \%$ vs. $14.7 \%, \mathrm{P}<0.001$ ). Absolute intake fell from $73.2 \mathrm{~g}$ to $60.9 \mathrm{~g}$ among $4-10 \mathrm{y}$ and from $81.6 \mathrm{~g}$ to $74.6 \mathrm{~g}$ among $11-18 \mathrm{y}$ olds $(\mathrm{P}<0.001)$. Many foods contributed to the reduction in NMES, but notably among $4-10 \mathrm{y}$, there were reductions in confectionery $(-6 \cdot 1 \mathrm{~g}$ NMES) and sugar-sweetened soft drinks $(-5.6 \mathrm{~g})$, partly offset by a rise in fruit juice $(+4.1 \mathrm{~g})$. Among $11-18 \mathrm{y}$ olds there were significant decreases in sugar from confectionery $(-5.8 \mathrm{~g})$, table sugar and spreads $(-2.5 \mathrm{~g})$ and cakes $(-2.0 \mathrm{~g})$, and a small rise in fruit juice $(+2.5 \mathrm{~g})$, but no significant change in sugar from soft drinks $(+1.4 \mathrm{~g})$.

\begin{tabular}{|c|c|c|c|c|c|c|c|c|c|c|}
\hline \multirow{2}{*}{ NMES g/day from source } & & \multicolumn{3}{|c|}{ 4-10 years } & \multicolumn{3}{|c|}{ 11-18 years } & \multicolumn{3}{|c|}{ Total 4-18 years } \\
\hline & & 1997 & $2008-12$ & $\mathrm{P}$ value & 1997 & $2008-12$ & $P$ value & 1997 & $2008-12$ & $P$ value \\
\hline & $\mathrm{N}$ & 835 & 803 & & 853 & 884 & & 1688 & 1687 & \\
\hline Soft drinks & Mean & $16 \cdot 8$ & $11 \cdot 2$ & $<0.001$ & $23 \cdot 6$ & 24.9 & $0 \cdot 412$ & $20 \cdot 2$ & $18 \cdot 8$ & $<0.001$ \\
\hline Fruit juice & Mean & $4 \cdot 7$ & 8.8 & $<0.001$ & $5 \cdot 3$ & $7 \cdot 8$ & 0.002 & 5 & $8 \cdot 2$ & $<0.001$ \\
\hline Chocolate confectionery & Mean & $7 \cdot 8$ & $4 \cdot 7$ & $<0.001$ & $10 \cdot 3$ & $6 \cdot 4$ & $<0.001$ & $9 \cdot 1$ & $5 \cdot 6$ & $<0.001$ \\
\hline Sugar confectionery & Mean & $8 \cdot 2$ & $5 \cdot 1$ & $<0.001$ & $6 \cdot 3$ & $4 \cdot 4$ & $<0.001$ & $7 \cdot 2$ & $4 \cdot 7$ & $<0.001$ \\
\hline Sugar and spreads & Mean & $5 \cdot 8$ & $4 \cdot 6$ & $<0 \cdot 001$ & $8 \cdot 6$ & $6 \cdot 1$ & $<0.001$ & $7 \cdot 2$ & $5 \cdot 4$ & $<0 \cdot 001$ \\
\hline Cakes \& pastries & Mean & 6 & $5 \cdot 1$ & $0 \cdot 04$ & $5 \cdot 7$ & $3 \cdot 8$ & $<0.001$ & $5 \cdot 9$ & $4 \cdot 4$ & $<0.001$ \\
\hline Biscuits & Mean & $5 \cdot 8$ & $4 \cdot 4$ & $<0 \cdot 001$ & $4 \cdot 7$ & $4 \cdot 7$ & $0 \cdot 001$ & $5 \cdot 2$ & $4 \cdot 6$ & $<0.001$ \\
\hline Breakfast cereals & Mean & $5 \cdot 8$ & $4 \cdot 0$ & $0 \cdot 002$ & $5 \cdot 3$ & $3 \cdot 8$ & $<0.001$ & $5 \cdot 5$ & 3.9 & $<0.001$ \\
\hline Puddings $\&$ ice cream & Mean & $4 \cdot 4$ & $3 \cdot 8$ & 0.005 & $3 \cdot 4$ & $2 \cdot 6$ & $<0.001$ & $3 \cdot 9$ & $3 \cdot 1$ & $<0.001$ \\
\hline \multirow[t]{2}{*}{ NMES from all food/ drink } & Mean & $73 \cdot 2$ & $60 \cdot 9$ & $<0 \cdot 001$ & $81 \cdot 6$ & $74 \cdot 6$ & $0 \cdot 001$ & 77.4 & $68 \cdot 5$ & $<0 \cdot 001$ \\
\hline & SE & 1 & $1 \cdot 5$ & & 1.4 & 2 & & $0 \cdot 8$ & $1 \cdot 3$ & \\
\hline \multirow[t]{2}{*}{$\%$ Energy from NMES } & Mean & $17 \cdot 4$ & $14 \cdot 7$ & $<0 \cdot 001$ & $16 \cdot 2$ & $15 \cdot 4$ & $0 \cdot 002$ & $16 \cdot 8$ & $15 \cdot 1$ & $<0.001$ \\
\hline & $\mathrm{SE}$ & $0 \cdot 2$ & $0 \cdot 3$ & & $0 \cdot 2$ & $0 \cdot 3$ & & $0 \cdot 1$ & $0 \cdot 2$ & \\
\hline
\end{tabular}

Whilst some progress is being made, on the current trajectory the new sugar target looks unachievable. A combination of strategies is needed including smaller portion sizes and reduced frequency of sugary foods and drinks, and in particular replacing sugar-sweetened drinks with low/ no sugar alternatives.

This study was funded by Sugar Nutrition UK

1. Public Health England (2015) Sugar Reduction: The evidence for action. https://www.gov.uk/government/publications/sugar-reductionfrom-evidence-into-action (accessed August 2016). 